\title{
Remote Sensing Techniques for Classification and Mapping of Sugarcane Growth
}

\author{
Marife Kung Villareal \\ School of Engineering, University of San Carlos, Cebu City, \\ Philippines and College of Engineering and Design, Silliman \\ University, Dumaguete City, Philippines \\ mkvillarea127@gmail.com
}

\author{
Alejandro Fernandez Tongco \\ School of Engineering \\ University of San Carlos \\ Cebu City, Philippines \\ aftongco@usc.edu.ph
}

\begin{abstract}
This study aimed to apply remote sensing technologies in delineating sugarcane (Saccharum officinarum) plantations and in identifying its growth stages. Considering the growing demand for sugarcane in the local and global markets, the need for a science-based resource inventory emerges. In this sense, remote sensing techniques' unique ability is vital to monitor crop growth and estimate crop yield. Object-Based Image Analysis (OBIA) concept was employed by utilizing orthophotos and Light Detection And Ranging (LiDAR) datasets. Specifically, the study applied the Support Vector Machine (SVM) algorithm to generate the resource map, validated by a handheld Global Positioning System (GPS). The classification result showed an accuracy of $98.4 \%$, delineating a total of 13.93 hectares of sugarcane plantation in the study area. The height information from LiDAR datasets aided in developing the rule-set that can further classify the sugarcane according to its growth stages. Results showed that the area distribution of sugarcane at establishment, tillering, yield formation, and ripening stage were $6.65 \%, 11.61 \%, 13.89 \%$, and $17.90 \%$ respectively. GPS validation points of the growth stages verified the accuracy of SVM. The accuracy results for growth stages, i.e. establishment, tillering, yield formation, and ripening are $88 \%, 94.4 \%, 96.3 \%$, and $91.7 \%$ respectively. The results proved the usefulness of SVM as a remote sensing classification technique which led to an exact mapping of the sugarcane areas as well as the practical use of LiDAR height information in estimating the growth stages of the mapped resource, both of which can provide valuable aid in estimating the potential sugarcane yield in the future.
\end{abstract}

Keywords-remote-sensing technologies; crop growth; remote images

\section{INTRODUCTION}

Accurate understanding and precise monitoring of land cover is essential for decision makers and producers in order to manage and improve crop production [1]. High value crops in Asia are divided into two types, the principal crops which are rice, wheat, soybeans, barley, corn and the cash crops which are tea, rubber, palm oil, coconut, and sugarcane [2]. In the Philippines, sugarcane is considered a high value crop, thus not surprisingly it is the fourth largest crop cultivated in the country yielding three major products: sugar, bio-ethanol, and power. The sugarcane is a plantation crop which requires big areas, greater than 10 hectares. The total area allocated to sugarcane planting nationwide totals to 422,384 hectares
(3.36\% of the country's arable land). The sugarcane industry provides employment to around 700,000 workers [3]. The country is the ninth largest sugar producer in the world and the second largest among the Association of South East Asian Nations (ASEAN) [4].

Sugarcane is an important perennial crop planted in tropical regions of many countries like Brazil, India, China, and the Philippines. Increased attention on sugarcane plantation in recent years is attributed to both the diminishing global sugar supply and on the growing demand for biomass energy as alternative to the fossil fuels commonly utilized today. In this case, sugarcane is an important resource of alcohol for biofuel. The sugarcane industry's potential impact on the Philippine economy and environmental aspects, calls for effective methods that can provide timely and accurate information on sugarcane growing areas as well as growth conditions at both regional and global scales [5]. Remote Sensing (RS) and Geographic Information System (GIS) provide useful tools for sustainable cultivation in developed countries' agriculture [5, 6]. RS techniques have been widely used in agriculture surveys in the past decades because of the unique ability of RS in monitoring crop growth and estimating crop yield with certain accuracy [7]. For instance, RS techniques give up-to-date and relatively right information for sugarcane crops management [8]. Studies have focused on various RS images to map sugarcane fields through automatic classification of Landsat images [9], through a rule-based classifier applied to SPOT image time series [10] or through an OBIA approach on high resolution remote sensing images time series [11] to categorize sugarcane fields into similar age units. On the other hand, GPS and GIS technologies are suitable for better management of land and other resources for sustainable crop production. Imagery could be integrated with other layers of spatial or geographical data in a GIS, with the use of GPS receivers to find positions of interest $[12,13]$.

The method employed in this study uses OBIA and the use of ideal features from LiDAR and Orthophoto data. The OBIA workflows are highly customizable allowing the integration of human semantics and hierarchical networks [14]. Generally, there are two main processes in OBIA, segmentation and classification. Segmentation is the process wherein adjacent pixels group together based on their homogeneity thereby 
creating meaningful "objects". These objects are then subjected to classification. Both segmentation and classification use different algorithms in eCognition with ease since much of the work referred to as OBIA originated from eCognition software [15-17]. Object-based classification is through user-defined rule-sets. The study also uses Support Vector Machine (SVM) which is a machine learning algorithm. SVM techniques can produce high classification accuracy and generate a neat image output [18]. Its most important characteristic is its ability to generalize well from a limited amount and/or quality of training data. Compared to other methods like Artificial Neural Networks (ANNs), SVM can yield comparable accuracy using a much smaller training sample size. This is due to the "support vector" concept that relies only on a few data points to define the hyper-plane that best separates the classes [19].

The advances in LiDAR technologies and the use of highresolution satellite images provide opportunities for detailed mapping and modelling of crops. In order to aid the precise inventory of sugarcane, this study utilizes LiDAR data, remote sensing techniques, and object-based image analysis to delineate sugarcane growth stages in the study area [20]. The LiDAR approach has emerged as a powerful active remote sensing method for direct measurement of $3 \mathrm{D}$ plant structure in precision agriculture $[21,22]$. LiDAR is valuable in the effort to improve identification and delineation of remotely-sensed data making it one of the better RS tools and techniques used in resource mapping. Numerous studies such as [23, 24] have proven that height features derived from LiDAR data can differentiate much between high and low vegetation. The highresolution aerial orthophotos and LiDAR data in the aforementioned studies address the problem of classification confusion caused by spectral mixing in areas with heterogeneous classes [1]. The LiDAR approach is an active RS technique like radar but uses laser light pulses to get elevation data [25]. The handheld GPS is also used for classification routine and for ground truthing and accuracy assessment of the growth stages and location of sugarcane crops. GPS technology has been adopted for better management of land and other resources for sustainable crop production [12]. These RS tools have been all utilized in this study. The sugar industry requires spatially explicit tools to provide reliable and precise information on the crops area and the field location to improve the accuracy in monitoring sugarcane production and yield estimates [26].

This study explores the application of RS techniques to map and monitor the sugarcane growth stages utilizing the classification algorithm coupled with LiDAR data and GPS technology for ground truth survey.

\section{METHODOLOGY}

\section{A. Study Area}

Tuburan is located $10^{\circ} 43^{\prime} 38.7^{\prime \prime}$ North and $123^{\circ} 49^{\prime} 55.4^{\prime \prime}$ East in the province of Cebu, Philippines. It has a total land area of 29,316 hectares. The study area for this mapping is Barangay Manga, Tuburan, Cebu. Manga is one of the villages in the town of Tuburan within the province of Cebu. The municipality has 7,329 hectares of land devoted to agriculture.
The main sources of income in Tuburan are agriculture and fishing. Sugarcane is among the cultivated crops.

\section{B. Sugarcane Growth Stages}

Mapping of sugarcane growth stages helps finding the locations of the newly planted, growing, and harvestable sugarcanes. Sugarcane crops that are ready for harvesting are properly monitored and yield production can be estimated. RS images have been used to map sugarcane fields [9] through an OBIA approach on high-resolution images and to categorize sugarcane fields into similar age units [11]. Sugarcane growth stages start from the establishment and tillering or germination phase. Establishment stage begins when two leaves appear on the stem. Tillering growth stage on the other hand, commences when the secondary sprouts forms from underground buds. This stage lasts for 4 to 6 months. The second stage is known as the vegetative and yield formation stage or the grand phase that will last for 5 to 8 months. Sugarcane vegetates during these months if properly supplied with heat and moisture, hence, this is also called the vegetative stage. The first two stages of sugarcane growth show the sugarcane in its young phase. Lastly, the ripening and maturation phases last for about three months. This growth stage is the harvestable sugarcane [27]. The use of technology like RS is necessary in the classification and identification of sugarcane growth, for instance authors in [28] suggested the need for automated methods to map sugarcane fields and other land uses.

\section{Process Workflow}

LiDAR data, GPS, orthophotos, and software like eCognition and QGIS were utilized for the classification map identifying the growth stages area of sugarcane crops. The LiDAR data show a high spatial resolution imagery which is effective in accurately mapping crop species by OBIA and for crop species classification [29-31]. The LiDAR derivatives and orthophotos have gone through a merging process and layer mixing to come up with a classified image (Figure 1).

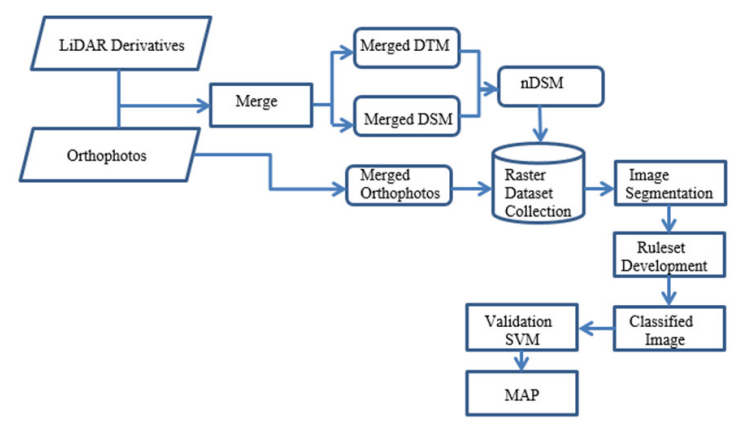

Fig. 1. Process workflow.

LiDAR derivative $\mathrm{nDSM}$ and orthophotos are required for crop classification. These LiDAR data give a $\mathrm{Z}$ or height information. The $\mathrm{nDSM}$ is the LiDAR height data normalized with respect to the ground surface. All ground points in $\mathrm{nDSM}$ are normalized to 0 . The LiDAR derivative produces $\mathrm{nDSM}$ by subtracting the Digital Terrain Model (DTM) from the canopy surface model or the Digital Surface Model (DSM). An orthophoto is an aerial RGB photo. Using the RGB alone, one 
can perform OBIA (segmentation and classification). The best feature in RGB is texture information by visual inspection. Using RGB orthophoto, estimation of classification accuracy and reliability is visually shown [32]. The nDSM and the merged orthophotos are now the raster data set collection needed for image segmentation and rule-set development in eCognition to produce the classified image.

\section{Segmentation}

This study uses OBIA for classification. Using OBIA, analysis and classifying of any raster image are possible. Segmentation uses eCognition software. Segmentation is the first step in OBIA. This is done so that instead of directly classifying pixels to produce a land cover or land classification result, classification is done by "segments" or groups of pixels ("object" based classification). Object-based image analysis uses four image layers. These four layers are: $\mathrm{nDSM}$ from LiDAR and Red, Green, Blue (RGB orthophoto) for presegmentation process. Figure 2 shows an orthophoto and an nDSM images of Barangay Mangga. These data are courtesy of Phil-LiDAR Research Center at the University of San Carlos Talamban Campus.

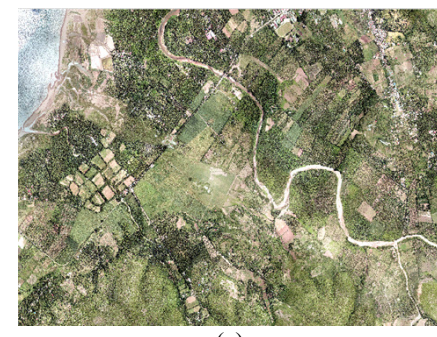

(a)

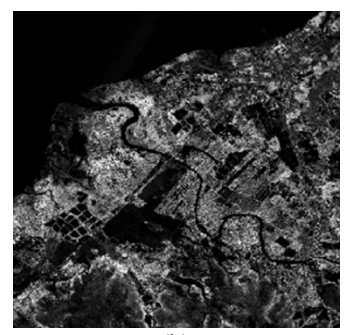

(b)
Fig. 2. (a) Orthophoto and (b) nDSM of Barangay Manga, courtesy of Phil-LiDAR Research Center at the University of San Carlos, Philippines.
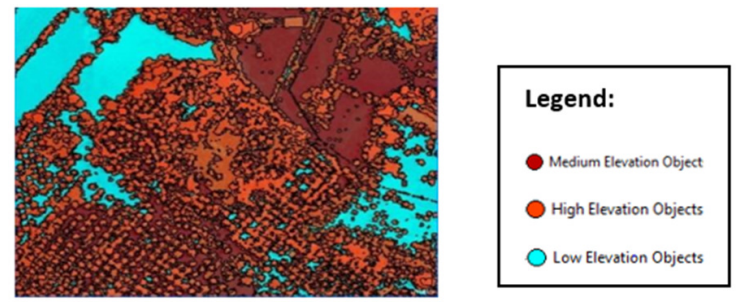

Fig. 3. Pre-segmentation process classifying high, medium, and low elevation objects using eCognition software.

The pre-segmentation process is done by using the eCognition software. The first segmentation process is quad tree-based segmentation using nDSM and having a scale parameter of $2.0 \mathrm{~m}$. Next is the spectral difference segmentation with most spectral difference of $2.0 \mathrm{~m}$. After the preclassification, follows the segmentation process where objects are assigned in high, medium or low elevation. High elevation objects have a mean threshold condition of mean nDSM greater than $2.0(\mathrm{nDSM}>2.0)$. Medium elevation objects have a mean threshold condition of mean $\mathrm{nDSM}$ less than or equal to 2.0 and greater than or equal to $0.25(0.25<=\mathrm{nDSM}<=2.0)$. The remaining are considered as low elevation objects. Figure 3 shows the classification result.

\section{E. Ground Survey}

Verification of the sugarcane crops and identification of the crops' growth stages was conducted by ground survey using a handheld GPS. In collecting data as points, it is advisable to collect multiple positions. The logging interval to select for collecting the positions can have an impact on accuracy. Collecting data at the point site for a longer time and then averaging positions, yields better results than collecting data at the point site for a shorter time. The summary of the validation points and the growth stages of sugarcane are shown in Table I.

TABLE I. AREA DISTRIBUTION OF VALIDATED SUGARCANE PLANTATIONS PER GROWTH STAGE.

\begin{tabular}{|c|c|}
\hline No. of validation points & Stages \\
\hline 8 & Establishment \\
\hline 12 & Tillering \\
\hline 19 & Yield formation \\
\hline 20 & Ripening \\
\hline
\end{tabular}

\section{F. Image Segmentation of Sugarcane and Growth Stages}

After pre-classification, the next step is the segmentation to capture the target subclasses which are the sugarcane and the shrub. SVM was employed to collect samples of sugarcane and shrub. Sugarcane is a medium elevation object. Segmentation setting for medium elevation and low elevation object is in a scale parameter of 40, 0.5 compactness, and 0.2 shape. The image layer weights are RGB. The threshold condition used for medium elevation objects is with a height of less than or equal to 2.0 and greater than or equal to 0.25 . Sugarcane and shrub were identified at this level: Sugarcane crops lie in the range of height of less than or equal to $2.0(<=2.0)$ and shrub and other species have a height that is greater than or equal to 0.25 . In Figure 4 the orthophoto and the classified output of sugarcane crops are shown.
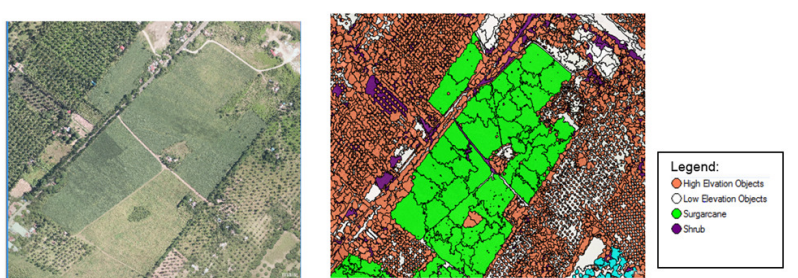

Fig. 4. Orthophoto and classified sugarcane crops. Data are courtesy of Phil-LiDAR Research Center at the University of San Carlos, Philippines.

The validation points taken per area of the growth stages of sugarcane by field survey are the basis to apply the rule-set for the segmentation process. In Figure 5 the orthophoto and the $\mathrm{nDSM}$ of the sugarcane growth stages can be seen.

\section{G. Supervised Learning Algorithm}

Sugarcane and shrub sample objects were extracted from the images. Extracted features from the collected samples were used for classification and for creating class definitions. Objects were subjected to a supervised learning algorithm to 
linearly separate classes and objects. Classification process utilized the SVM algorithm, whose most important characteristic is its ability to generalize from a limited amount and or quality of training data [19,33]. SVM is employed to classify land features in the images. The classified objects are acquired from the segmentation procedure. The LiDAR derivative (nDSM) is a feature for developing the SVM model. (a)

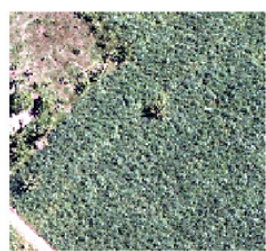

(b)

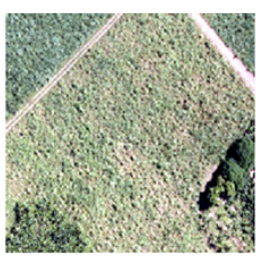

(c)

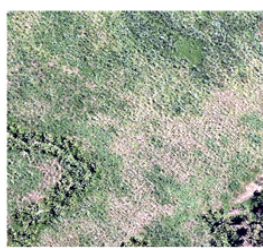

(d)

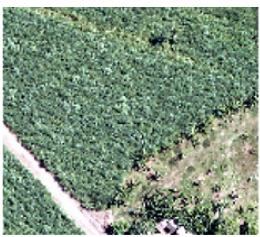

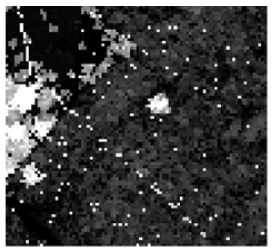
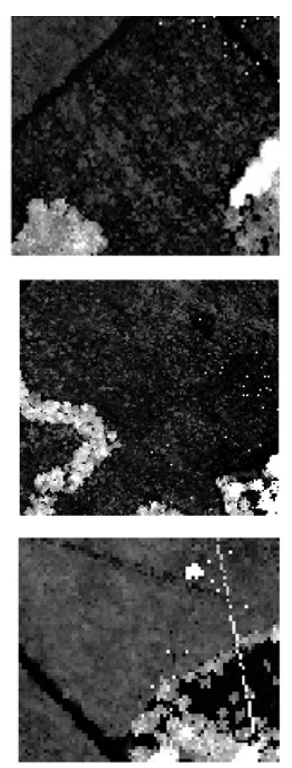

Fig. 5. Growth stages of sugarcane: (a) Establishment, (b) Tillering, (c) Yield formation, (d) Ripening. (a) and (b) are courtesy of Phil-LiDAR Research Center at the University of San Carlos, Philippines.

\section{RESULTS AND DISCUSSION}

\section{A. Classified Image}

In Figure 6 we can see the classified image of sugarcane while Table II shows the accuracy assessment result.

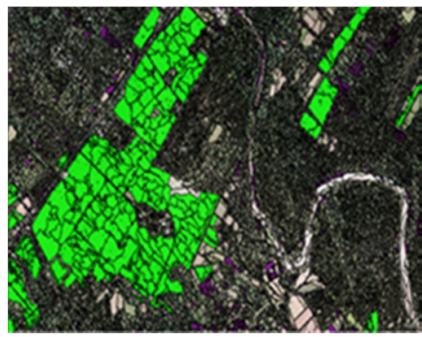

Fig. 6. Result of the SVM process using eCognition software. Data are courtesy of Phil-LiDAR Research Center at the University of San Carlos, Philippines.
TABLE II. SVM'S ACCURACY ASSESSMENT FOR SUGARCANE

\begin{tabular}{|c|c|c|c|}
\hline \multicolumn{5}{|c|}{ Confusion matrix } \\
\hline User class/Sample & Sugarcane & Shrub & Sum \\
\hline Sugarcane & 134 & 497 & 631 \\
\hline Shrub & 30 & 109771 & 111083 \\
\hline Sum & 1446 & 110268 & \\
\hline \multicolumn{4}{|c|}{ Accuracy } \\
\hline Producer & 0.09266943292 & 0.9954928 & \\
\hline User & 0.2123613 & 0.9881890 & \\
\hline Hellden & 0.129 & 0.9918275 & \\
\hline Short & 0.06896551724 & 0.9837874 & \\
\hline KIA per class & 0.08751539866 & 0.202 & \\
\hline \multicolumn{4}{|c|}{ Totals } \\
\hline Overall accuracy & 0.9838069 & \\
\hline KIA & 0.1221280 & & \\
\hline
\end{tabular}

Identification of the growth stages was conducted by GPS field survey. The best features used in RGB are texture information by visual inspection. According to [32] the classification accuracy and reliability are visually estimated using RGB orthomosaic. With the validation points of the GPS, the classification process using SVM parameters was applied to these areas. The high accurate results in growth stages classification can then be used for mapping and monitoring sugarcane growth stages in Philippines and in other sugarproducing countries in the world. The classified images of each growth stage are shown in Figure 7. Table III shows the accuracy assessment result of each stage.

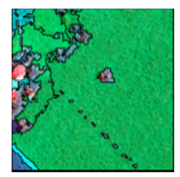

(a)

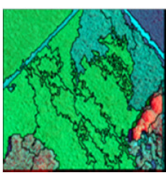

(b)

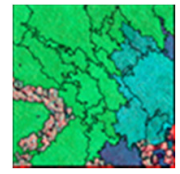

(c)

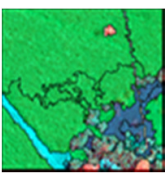

(d)
Fig. 7. Classified images of the growth stages of sugarcane, processed with eCognition software. (a) Establishment stage, (b) tillering stage, (c) yield formation stage, and (d) ripening stage. Data is courtesy of Phil-LiDAR Research Center at the University of San Carlos, Philippines.

TABLE III. ACCURACY ASSESSMENT AFTER SVM

\begin{tabular}{|c|c|c|}
\hline Growth Stages & Accuracy assessment (\%) & KIA (\%) \\
\hline Establishment & 87.5 & 75 \\
\hline Tillering & 94.4 & 83.9 \\
\hline Yield formation & 96.3 & 88.6 \\
\hline Ripening & 91.7 & 82.3 \\
\hline
\end{tabular}

The land classification map of the growth stages of sugarcane is exhibited in Figure 8. The points were overlaid and merged with the shape file of Barangay Manga using QGIS to find the growth stages of sugarcane. Utilization of QGIS allowed the feature selection of the specific image map of the area.

\section{B. Comparison}

The research in [34] on monitoring sugarcane growth phases based on satellite images is more or less similar to the current study, though the data employed in [34] come from Landsat 8, and the vegetation indices of Normalized Difference Vegetation Index (NDVI) and Enhanced Normalized Difference Vegetation Index (ENDVI) are employed to analyze the growing phases. Field survey was also conducted. The 
NDVI and ENDVI results confirm the field data verification [34]. In the current study, the RS technologies used were LiDAR data, orthophoto images, and handheld GPS for field survey. The results proved that they are also useful in the classification and identification of sugarcane growth stages. Both studies use RS approaches in analyzing the growing phases of sugarcane and the harvest of sugarcane crops.

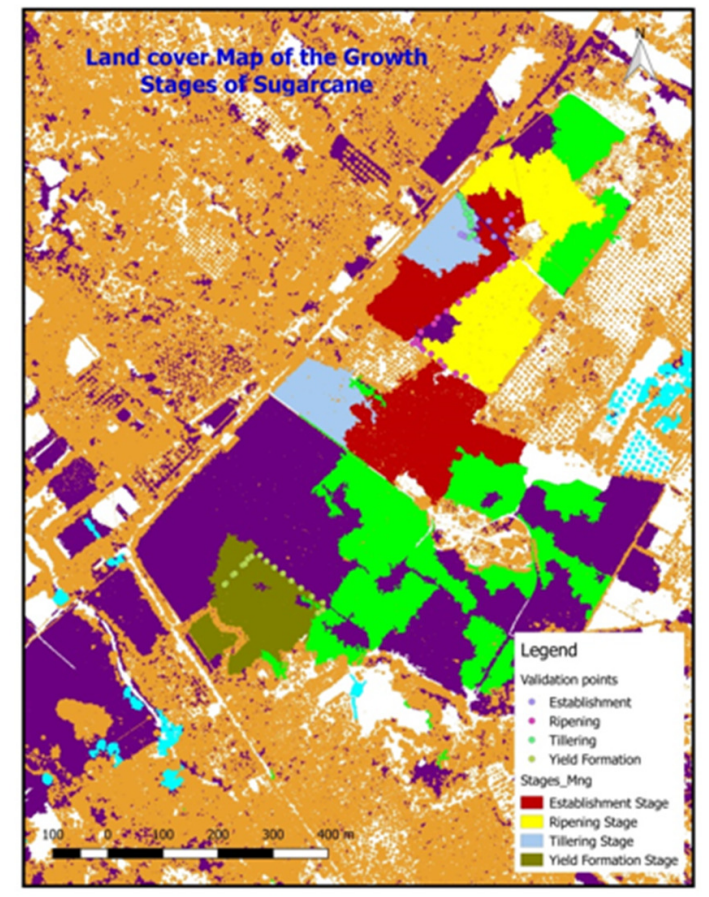

Fig. 8. Land cover map of the growth stages of sugarcane processed with QGIS software. Data are courtesy of Phil-LiDAR Research Center at the University of San Carlos, Philippines.

\section{CONCLUSION}

This study was able to test remote sensing technology and techniques in classifying sugarcane crop growth stages. eCognition and QGIS software, LiDAR data, orthophotos, field work, and ground survey, all contributed to the making of the classification map, an output that locates the area of sugarcane plants and the specific growth stages of the crops in the study area. Sugarcane plant classification revealed a high accuracy result of $98.4 \%$ while the ripening stage classification had an accuracy of $91.7 \%$. The other growth stages, namely establishment, tillering, and yield formation yielded accuracy rates of $88 \%, 94.4 \%$, and $96.3 \%$ respectively. With this highly satisfactory accuracy assessment, the rule set developed in mapping and identifying of the growth stages of sugarcane crops in this study could be the basis in mapping and checking other areas of sugarcane plantation and their growth stages in other parts of the country.

\section{ACKNOWLEDGEMENT}

This research was supported by Phil-LiDAR Research Center at the University of San Carlos, Talamban Campus through its Project Leader Dr. Roland Emerito Otadoy, Research Associate Engr. Beethoven Arellano, and Mr. James
Earl Cabillas of Phil-LiDAR 2. We would also wish to thank Silliman University for providing the financial support.

\section{REFERENCES}

[1] R. Candare, M. Japitana, J. E. Cubillas, and C. B. Ramirez, "Mapping of High Value Crops Through an Object-Based SVM Model Using LiDAR Data and Orthophoto in Agusan del Norte, Philippines," ISPRS Annals of Photogrammetry, Remote Sensing and Spatial Information Sciences, vol. III-7, pp. 165-172, Jun. 2016, doi: 10.5194/isprs-annals-III-7-1652016.

[2] “Asia - Agriculture," Encyclopedia Britannica. https://www.britannica. com/place/Asia (accessed Jul. 11, 2020).

[3] "Sugar industry of the Philippines," Wikipedia. Mar. 19, 2020, Accessed: Jul. 11, 2020. [Online]. Available: https://en.wikipedia.org/w/index.php?title=Sugar_industry_of_the_Phili ppines\&oldid=946332977.

[4] S. Solomon, M. Swapna, X. Vo-Tong, and Y. Mon, "Development of Sugar Industry in ASEAN Countries," Sugar Tech, vol. 18, Nov. 2016, doi: 10.1007/s12355-016-0497-z.

[5] H. Lin, J. Chen, Z. Pei, S. Zhang, and X. Hu, "Monitoring Sugarcane Growth Using ENVISAT ASAR Data," IEEE Transactions on Geoscience and Remote Sensing, vol. 47, no. 8, pp. 2572-2580, Aug. 2009, doi: 10.1109/TGRS.2009.2015769.

[6] M. S. Hassan and S. Mahmud-ul-islam, "Rapid Identification of the Sugarcane Cultivation Area and Crop Growth Condition at Ishwardi Upazila, Bangladesh Using Landsat Imagery," International Journal of Research and Review, vol. 2, no. 4, pp. 162-168, 2015.

[7] E. M. Abdel-Rahman and F. B. Ahmed, "The application of remote sensing techniques to sugarcane (Saccharum spp. hybrid) production: a review of the literature," International Journal of Remote Sensing, vol. 29, no. 13, pp. 3753-3767, Jun. 2008, doi: $10.1080 / 01431160701874603$.

[8] B. F. T. Rudorff, D. A. Aguiar, W. F. Silva, L. M. Sugawara, M. Adami, and M. A. Moreira, "Studies on the Rapid Expansion of Sugarcane for Ethanol Production in São Paulo State (Brazil) Using Landsat Data," Remote Sensing, vol. 2, no. 4, pp. 1057-1076, Apr. 2010, doi: 10.3390/rs2041057.

[9] M. El Hajj, A. Bégué, S. Guillaume, and J.-F. Martiné, "Integrating SPOT-5 time series, crop growth modeling and expert knowledge for monitoring agricultural practices - The case of sugarcane harvest on Reunion Island," Remote Sensing of Environment, vol. 113, no. 10, pp. 2052-2061, Oct. 2009, doi: 10.1016/j.rse.2009.04.009.

[10] M. A. Vieira, A. R. Formaggio, C. D. Rennó, C. Atzberger, D. A. Aguiar, and M. P. Mello, "Object Based Image Analysis and Data Mining applied to a remotely sensed Landsat time-series to map sugarcane over large areas," Remote Sensing of Environment, vol. 123, pp. 553-562, Aug. 2012, doi: 10.1016/j.rse.2012.04.011.

[11] C. Palaniswami, P. Gopalasundaram, and A. Bhaskaran, "Application of GPS and GIS in Sugarcane Agriculture," Sugar Tech, vol. 13, no. 4, pp. 360-365, Dec. 2011, doi: 10.1007/s12355-011-0098-9.

[12] T. Blaschke, S. Lang, D. Tiede, M. Papadakis, and A. Györi, "ObjectBased Image Analysis Beyond Remote Sensing - the Human Perspective," ISPRS - International Archives of the Photogrammetry, Remote Sensing and Spatial Information Sciences, vol. 41B7, pp. 879882, Jun. 2016, doi: 10.5194/isprs-archives-XLI-B7-879-2016.

[13] U. C. Benz, P. Hofmann, G. Willhauck, I. Lingenfelder, and M. Heynen, "Multi-resolution, object-oriented fuzzy analysis of remote sensing data for GIS-ready information," ISPRS Journal of Photogrammetry and Remote Sensing, vol. 58, no. 3, pp. 239-258, Jan. 2004, doi: 10.1016/j.isprsjprs.2003.10.002.

[14] D. Flanders, M. Hall-Beyer, and J. Pereverzoff, "Preliminary evaluation of eCognition object-based software for cut block delineation and feature extraction," Canadian Journal of Remote Sensing, vol. 29, no. 4, pp. 441-452, Jan. 2003, doi: 10.5589/m03-006.

[15] M. Baatz and A. Schäpe, "Multiresolution Segmentation: an optimization approach for high quality multi-scale image segmentation," presented at the Beutrage zum AGIT-Symposium, Salzburg, Heidelberg, 2000 . 
[16] R. Devadas, R. J. Denham, and M. Pringle, "Support Vector Machine Classification of Object-based Data for Crop Mapping, Using Multitemporal Landsat Imagery," in International Archives of the Photogrammetry, Remote Sensing and Spatial Information Sciences, Melbourne, Australia, Sep. 2012, vol. XXXIX-B7, pp. 185-190, doi: 10.5194/isprsarchives-XXXIX-B7-185-2012.

[17] G. Mountrakis, J. Im, and C. Ogole, "Support vector machines in remote sensing: A review," ISPRS Journal of Photogrammetry and Remote Sensing, vol. 66, no. 3, pp. 247-259, May 2011, doi: 10.1016/j.isprsjprs.2010.11.001.

[18] M. V. Japitana, J. E. D. Cubillas, and A. G. Apdohan, "Coupling LiDAR Data and LANDSAT 8 OLI in Delineating Corn Plantations in Butuan City, Philippines," presented at the 36th Asian Conference on Remote Sensing, Quezon City, Metro Manila, Philippines, Oct. 2015

[19] W. S. Lee, V. Alchanatis, C. Yang, M. Hirafuji, D. Moshou, and C. Li, "Sensing technologies for precision specialty crop production," Computers and Electronics in Agriculture, vol. 74, no. 1, pp. 2-33, Oct. 2010, doi: 10.1016/j.compag.2010.08.005.

[20] J. R. Rosell et al., "Obtaining the three-dimensional structure of tree orchards from remote 2D terrestrial LIDAR scanning," Agricultural and Forest Meteorology, vol. 149, no. 9, pp. 1505-1515, Sep. 2009, doi: 10.1016/j.agrformet.2009.04.008.

[21] A. P. Charaniya, R. Manduchi, and S. K. Lodha, "Supervised Parametric Classification of Aerial LiDAR Data," in 2004 Conference on Computer Vision and Pattern Recognition Workshop, Jun. 2004, pp. 30-30, doi: 10.1109/CVPR.2004.446.

[22] Y. Huang et al., "Toward automatic estimation of urban green volume using airborne LiDAR data and high resolution Remote Sensing images," Frontiers of Earth Science, vol. 7, no. 1, pp. 43-54, Mar. 2013, doi: 10.1007/s1 1707-012-0339-6.

[23] J. Carter et al., Lidar 101: An Introduction to Lidar Technology, Data, and Applications. Charleston, SC, USA: National Oceanic and Atmospheric Administration (NOAA) Coastal Services Center, 2012.

[24] FAS/Nairobi Staff, "Sugar Annual Kenya Sugar Annual Report," USDA FAS, Nairobi, Kenya, Apr. 2012.

[25] T. Blaschke, "Object based image analysis for remote sensing," ISPRS Journal of Photogrammetry and Remote Sensing, vol. 65, no. 1, pp. 216, Jan. 2010, doi: 10.1016/j.isprsjprs.2009.06.004.

[26] P. S. Thenkabail and Z. Wu, "An Automated Cropland Classification Algorithm (ACCA) for Tajikistan by combining Landsat, MODIS, and secondary data," Remote Sensing, vol. 4, no. 10, pp. 2890-2918, 2012.

[27] D. C. Duro, S. E. Franklin, and M. G. Dubé, “A comparison of pixelbased and object-based image analysis with selected machine learning algorithms for the classification of agricultural landscapes using SPOT-5 HRG imagery," Remote Sensing of Environment, vol. 118, pp. 259-272, Mar. 2012, doi: 10.1016/j.rse.2011.11.020.

[28] J. M. Peña-Barragán, M. K. Ngugi, R. E. Plant, and J. Six, “Objectbased crop identification using multiple vegetation indices, textural features and crop phenology," Remote Sensing of Environment, vol. 115, no. 6, pp. 1301-1316, Jun. 2011, doi: 10.1016/j.rse.2011.01.009.

[29] C. Conrad, S. Fritsch, J. Zeidler, G. Rücker, and S. Dech, "Per-Field Irrigated Crop Classification in Arid Central Asia Using SPOT and ASTER Data," Remote Sensing, vol. 2, no. 4, pp. 1035-1056, Apr. 2010 , doi: $10.3390 / \mathrm{rs} 2041035$

[30] O. Nevalainen et al., "Individual Tree Detection and Classification with UAV-Based Photogrammetric Point Clouds and Hyperspectral Imaging," Remote Sensing, vol. 9, no. 3, p. 185, Mar. 2017, doi: $10.3390 / \mathrm{rs} 9030185$.

[31] G. M. Foody and A. Mathur, "Toward intelligent training of supervised image classifications: directing training data acquisition for SVM classification,” Remote Sensing of Environment, vol. 93, no. 1, pp. 107 117, Oct. 2004, doi: 10.1016/j.rse.2004.06.017.

[32] T. M. Susantoro, K. Wikantika, A. B. Harto, and D. Suwardi, "Monitoring Sugarcane Growth Phases Based on Satellite Image Analysis (A Case Study in Indramayu and its Surrounding, West Java, Indonesia)," HAYATI Journal of Biosciences, vol. 26, no. 3, pp. 117 117, Dec. 2019, doi: 10.4308/hjb.26.3.\%x
[33] D. Pylarinos and I. Pellas, "Incorporating Open/Free GIS and GPS Software in Power Transmission Line Routine Work: The Case of Crete and Rhodes," Engineering, Technology \& Applied Science Research, vol. 7, no. 1, pp. 1316-1322, Feb. 2017.

[34] M. V. Japitana and M. E. C. Burce, "A Satellite-based Remote Sensing Technique for Surface Water Quality Estimation," Engineering, Technology \& Applied Science Research, vol. 9, no. 2, pp. 3965-3970, Apr. 2019. 